\title{
Perioperative outcomes and mid-term effects in performing video- assisted thoracoscopic extended thymectomy for myasthenia gravis: subxiphoid versus right thoracic approaches
}

\author{
Zhihong Qiu", Liru Chen", Qin Lin, Hao Wu, Huangtao Sun, Xin Zhou, Yeji Hu, Lei Peng, \\ Yangchun Liu, Quan Xu
}

Department of Cardiothoracic Surgery, Jiangxi Provincial People's Hospital Affiliated to Nanchang University, Nanchang 330006, China

Contributions: (I) Conception and design: Y Liu, Q Xu; (II) Administrative support: Y Liu, Q Xu; (III) Provision of study materials or patients: All authors; (IV) Collection and assembly of data: Z Qiu, L Chen; (V) Data analysis and interpretation: Z Qiu, L Chen; (VI) Manuscript writing: All authors; (VII) Final approval of manuscript: All authors.

\#These authors contributed equally to this work.

Correspondence to: Yangchun Liu, MD. Department of Cardiothoracic Surgery, Jiangxi Provincial People's Hospital Affiliated to Nanchang University, Nanchang 330006, China. Email: lyc195411@sina.com; Quan Xu, MD. Department of Thoracic Surgery, Jiangxi Provincial People’s Hospital Affiliated to Nanchang University, Nanchang 330006, China. Email: xuquan7210@163.com.

Background: Approaches of thoracoscopic thymectomy for myasthenia gravis (MG) are debatable. The subxiphoid approach is widely utilized recent years for its better visualization of the anterior mediastinum. In the present study, we compared perioperative outcomes and mid-term effects of the extended thymectomy for MG between the subxiphoid approach and the routine right-thoracic approach.

Methods: One hundred and thirty-one MG patients treated with thoracoscopic extended thymectomy were analyzed. Among them, 68 patients were operated on via the subxiphoid approach and the other 63 via the conventional right-side unilateral approach. The patient outcomes were retrospectively reviewed and evaluated. Mid-term clinical outcome was assessed according to the classification system proposed by the Myasthenia Gravis Foundation of America (MGFA). Clinical efficacy and variables influencing outcome were evaluated by the Kaplan-Meier method and Cox proportional hazards regression analysis.

Results: Compared with the right thoracic approach, the duration of the procedure via the subxiphoid approach was significantly shorter $(\mathrm{P}=0.035)$, the rates of total thymectomy were higher $(\mathrm{P}=0.028)$, and the pain scores on postoperative days 1,3 , and 7 were significantly lower $(\mathrm{P}<0.001, \mathrm{P}<0.001$, and $\mathrm{P}=0.03$, respectively). A total of 112 patients with $\mathrm{MG}$ were followed up. The subxiphoid approach group reported higher rates of complete stable remission (CSR) and effective treatment of MG, although these differences were not statistically significant $(\mathrm{Z}=-0.484, \mathrm{P}=0.627)$. By multivariate Cox proportional hazards modes analysis, the chance of CSR was significantly increased when age <40 (OR: 2.623, 95\% CI: 1.150-5.983, $\mathrm{P}=0.022$ ), non-thymomatous MG (OR: $1.078,95 \% \mathrm{CI}: 1.101-3.316, \mathrm{P}=0.021$ ) and MGFA clinical classification (OR: 2.024, 95\%:1.164-3.523, $\mathrm{P}=0.013$ ).

Conclusions: The subxiphoid approach has shorter operation time, higher rates of total thymectomy and better quality of life compared with the lateral thoracoscopic approach. Preoperative age, pathological diagnoses and MGFA Clinical Classification are the independent risk factors for non-complete stable remission (NCSR) after thymectomy.

Keywords: Myasthenia gravis (MG); subxiphoid; extended thymectomy

Submitted Dec 24, 2019. Accepted for publication Feb 28, 2020.

doi: $10.21037 /$ jtd.2020.03.43

View this article at: http://dx.doi.org/10.21037/jtd.2020.03.43 


\section{Introduction}

Thymectomy for myasthenia gravis (MG) is now recognized as a standard method of therapy combined with medical management $(1,2)$. In recent years, multiple studies have been published, confirming that minimally invasive techniques of thymectomy were effective therapy for the treatment of MG $(1,3)$. There are several surgical approaches for minimally invasive thymectomy, including conventional unilateral (right-or-left-sided) video-assisted thoracic surgery (VATS), bilateral VATS, robotic videoassisted techniques (RATS), transcervical thymectomy or combination strategies. Some approaches have been designed and optimized for thoracoscopic thymectomy, especially for extended thymectomy, VATS has become a routine technique for extended thymectomy (4-6). However, there are still some shortcomings $(7,8)$. The conventional unilateral (VATS) approach may lead to inadequate exposure of the contralateral pleural cavity. The use of bilateral thoracoscopy may lead to intercostal pain and too many thoracic port sites. Transcervical thymectomy may lead to inadequate exposure of the lower pleural cavity. To work around these issues, we recently performed extended thymectomy using the subxiphoid approach that can improve visualization of the anterior mediastinum and facilitate dissection up to the thyroid possible (9). Thus complete removal of the thymus, even the resection of invaded tissues, the fat tissues near the cardiacdiaphragmatic angles and the upper poles of the thymus, could be performed more easily (10). Studies have shown that the mid-term efficacy of MG patients is also closely related to the ectopic thymus tissue $(11,12)$. The main principle of extended thymectomy for MG is the complete removal of the entire thymic gland.

The thymectomy under the subxiphoid approach as a surgical procedure has been reported abroad for a long time $(13,14)$. During the last decade, the subxiphoid approach was used successfully. However, earlier studies have been limited by small patient populations and lack of long-term follow-up; existing reports rarely supply long-term followup results. In the present retrospective study, we compared the clinical effects and long-term follow-up outcomes of the two distinctly different operative techniques in the management of MG, and to provide theoretical reference for the subxiphoid approach in MG surgery.

\section{Methods}

\section{Patients choice}

We retrospectively analyzed the clinical data of 131 cases of MG treated by subxiphoid or right-side approach VATS in the department of Cardiothoracic Surgery, Jiangxi Provincial People's Hospital from January 2013 to October 2017. Among them, 68 patients were operated via the subxiphoid approach and the other 63 via the conventional right-side unilateral approach. The Institutional Review Board of the hospital approved the study, and written informed consent from each patient was obtained before the procedure. Case inclusion criteria: (I) preoperative diagnosis of MG based on clinical symptoms, the Tensilon test, electrophysiological test, and serological test: detection of anti-acetylcholine-receptor antibodies (AchR-Ab) (15); (II) diagnosis of tumor by using chest computed tomography (CT), The tumor location was carefully assessed to make sure there was no major invasion to the trachea, the heart, the aorta, and other big vessels; (III) preoperative comprehensive evaluation of surgical tolerance, no obvious surgical contraindications. Exclusion criteria: (I) thymoma receiving radiotherapy and chemotherapy before surgery; (II) recurrence after thymoma resection; (III) thymoma diameter $>5 \mathrm{~cm}$, aggressive thymoma is expected to require resection of large vessels and artificial blood vessels reconstuction; (IV) incomplete case data.

\section{Preoperative preparation}

Preoperative preparation of the MG patient included drugs (anticholinesterase, steroid, immunosuppressive, immunoglobulins, etc.) and plasmapheresis. All patients were treated with pyridostigmine and prednisone to improve MG symptoms and obtain preoperational safety. The postoperative crisis has been related to a history of myasthenic crisis, severity (type IIA and above according to the clinical grade of osserman classification) (15), preoperative presence of bulbar weakness $(15,16)$, preoperative AchR-Ab serum levels of $>100 \mathrm{nmol} / \mathrm{L}$ (16-18). For these patients, plasmapheresis and intravenous immunoglobulin maintenance therapy can be considered. For patients with MG of Osserman type IIA and above, surgery should be performed after careful preoperative management is conducted, so as to prevent a postoperative myasthenic crisis. 

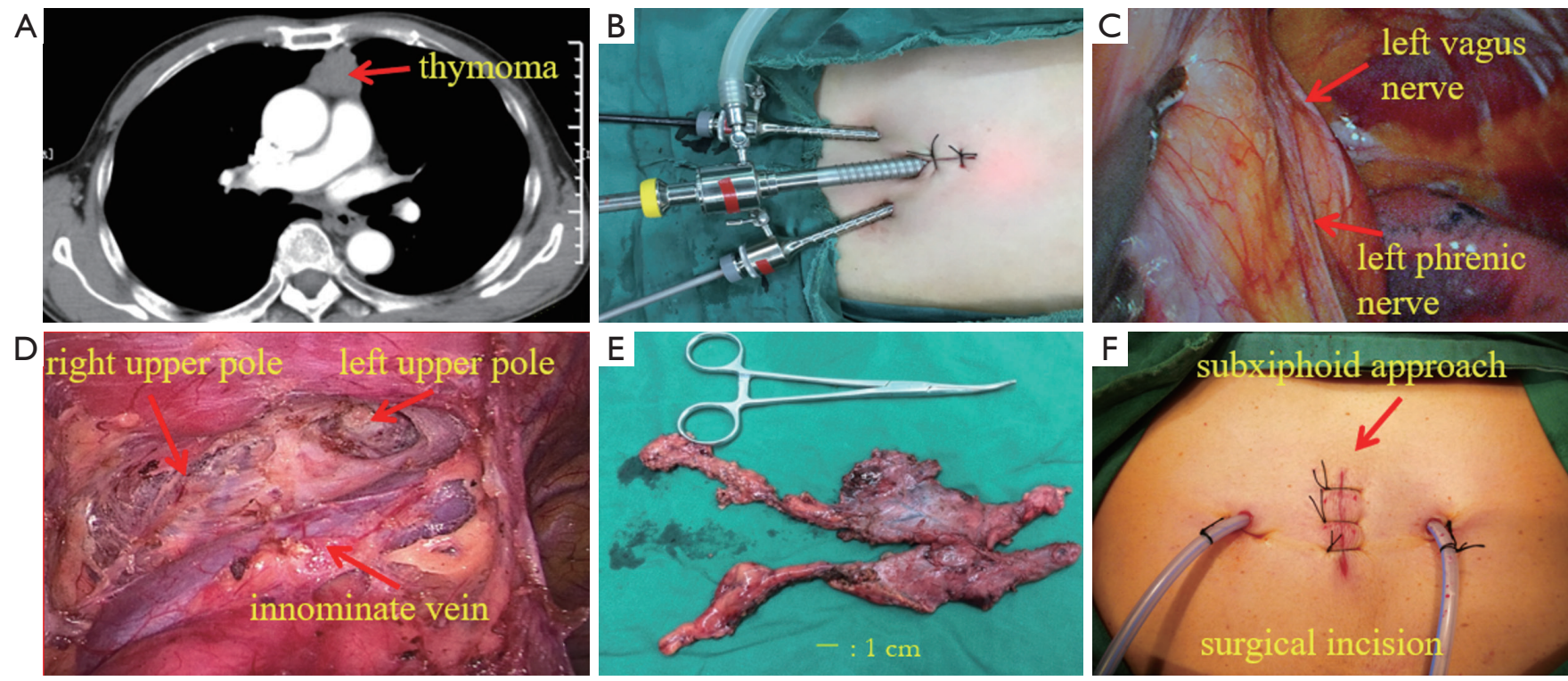

Figure 1 Preoperative CT, surgical incision, intraoperative view under subxiphoid approach and the specimen. (A) Preoperative CT showed thymoma, $3.5 \mathrm{~cm} \times 3.3 \mathrm{~cm}$; (B) incision designed for subxiphoid approach assisted with $\mathrm{CO}_{2}$ artificial pneumothorax; (C) left phrenic nerve during surgery; (D) anterior mediastinum after extended thymectomy; (E) specimen of completely resected thymus and bilateral mediastinal adipose tissue; (F) surgical incision after suture.

\section{Surgical technique}

Subxiphoid approach: All 68 patients underwent singlelumen endotracheal intubation and general anesthesia. The patient was placed in the supine position on the operating table with the legs open. The surgeon stood between the patient's legs, and the assistant was on the left or right side. A 2-cm straight incision was made in the middle of the xiphoid process, the incision was used as a thoracoscope hole, and the xiphoid process could be removed if necessary. The surgeon used the oval forceps to release the left and right soft tissue gaps behind the sternum, from the anterior mediastinum tunnel. Two $0.5 \mathrm{~cm}$ extrapleural thoracic ports under the bilateral costal arches were created, and this incision was used as an operation hole. The thread puncture cone with a diameter of $0.5 \mathrm{~cm}$ was placed under the guidance of the finger. A $2-\mathrm{cm}$ diameter puncture cone was placed in the incision under the xiphoid process, placed in a thoracoscope, connected to a carbon dioxide $\left(\mathrm{CO}_{2}\right)$ pneumoperitoneum, and the pressure was set to $1.330-1.596 \mathrm{KPa}(10-12 \mathrm{mmHg})$ to form an artificial pneumothorax, which maintains the lung and diaphragm off the surgical field to ensure a sufficient surgical field at the anterior mediastinum. Place the grasping forceps inside the puncture cone on the lower edge of the right rib arch and place the ultrasonic knife on the left side. The surgeon used an ultrasound scalpel to cut the left and right mediastinum pleura, cleared the right pericardium fat, and opened the phrenic nerve 0.5 -cm upward to separate the right thymus. The thymus artery and vein were cut with an ultrasound scalpel, and the upper thymus was separated. The same method was used to remove the left pericardium fat, separate the left thymus gland, altogether remove the thymus and mediastinal fat. The specimen bag is placed in the right thoracic cavity through the incision under the xiphoid process, the thymus and adipose tissue are taken out, and the left and right thoracic drainage tubes are placed through the incision of the lower edge of the rib arch (Figure 1).

Right-side approach: all 63 patients underwent doublelumen endotracheal intubation, single-lung ventilation, and general anesthesia.

Use the left supine position, and the right upper limb is suspended from the head frame. The observing port was created at the right axillary midline line in the fourth intercostal space, and the other two ports were made as to the operation hole along the anterior axillary lines in the third and fifth intercostal spaces. With the right internal mammary vessel as a marker, the right mediastinal pleura 
was cut with an ultrasonic scalpel along the right internal mammary vessel, up to the internal mammary vein enters the right subclavian vein and down to the diaphragm. The right lower pole of the thymus, the pre-pericardial fat, and the right phrenic nerve fat were dissected after the fat and thymus are removed. The fat near the left phrenic nerve and left cardiac-diaphragmatic angle was removed as much as possible by similar methods. Expand the operating hole to remove the thymus. The chest drainage tube was placed through the incision of the sixth intercostal space.

\section{Postoperative management and evaluation}

After surgery, all patients were managed according to the same protocol. In principle, all the patients resumed their preoperative medications for the control of MG, but the critical patients adjust the dosage and time of pyridostigmine and prednisone according to postoperative clinical symptoms, such as saliva, sputum, and autonomic respiratory status. In the past, we have emphasized the use of pyridostigmine enough. Clinical observations have revealed an abnormal increase in oral and respiratory secretions in the critical patients because of the cholinergic effect of salivary glands in the target organ, and patients with cough, swallowing weakness, easy to lead to airway obstruction and respiratory disorders. Since 2006, we have used low dose pyridostigmine to alleviate postoperative cholinergic effects, combined with plasmapheresis in patients with severe $M G$, and this method was confirmed to be safe and reliable.

For each patient, a retrospective case note review including the following variables was done: age at the time of surgery, gender, maximal tumor diameter, Myasthenia Gravis Foundation of America (MGFA) clinical classification, pathological diagnoses, operation times, blood loss during operation, rate of conversion to thoracotomy, duration of chest drainage, postoperative delayed extubation, Total drainage, hospital stay after operation, postoperative complications, postoperative pain, massive bleeding needed blood transfusion, extent of resection, 30-day mortality. Among them, the operation time starts from the skin cutting and closes each operation hole as the endpoint; postoperative delayed extubation was defined as a total postoperative mechanical ventilation support time of $>24$ hours in the immediate postoperative period. The chest drainage tubes can be removed when the drainage volume is $<50 \mathrm{~mL}$. Researches show that postoperative myasthenic patients in whom extubation has been delayed more than 24 hours also should be considered crisis (15), so defined as delayed postoperative extubation ( $>24$ hours). The postoperative pain was evaluated by using the Visual Analog Pain Score (VAS-score) at 24, 72 hours operation and 7 days after operation.

Mid-term follow-up was obtained in 112 (85.5\%) of 131 patients by both outpatient service and telephone. The patients were followed up for one time in the first month after surgery, followed by two times every six months, and then followed up once a year for evaluation of efficacy, obtaining and recording relevant clinical information, and guiding patients to postoperative treatment. According to the American MGFA (19), the efficacy of the MG score was evaluated: (I) treatment is effective, including complete stable remission (CSR), pharmacological remission (PR), and minor symptom changes (minor manifestation, $\mathrm{MM}$ ); improved (I); (II) treatment is ineffective, including no change $(\mathrm{U})$, worse $(\mathrm{W})$, exacerbation $(\mathrm{E})$, death (death, $\mathrm{D})$. Except for CSR, all the other results are non-complete stable remission (NCSR). The number of total thymectomy and partial thymectomy were counted. Total thymectomy and partial thymectomy were counted in each group, and total thymectomy was defined as the resection range, including complete upper and lower thymic poles in addition to the tumor and surrounding thymic and adipose tissue. Partial thymectomy is defined as the removal of the tumor and surrounding parts of the thymus and adipose tissue.

\section{Statistical analysis}

For statistical analysis, we used SPSS for Windows, version 22 of IBM SPSS Statistics software, and the results were described as frequencies with percentages for categorical variables, means with standard deviations (SDs) for normally distributed continuous variables, or medians with interquartile ranges for non-normally distributed continuous variables. Chi-squared test, one-way analysis of variance (ANOVA). Cox regression analysis was used for multivariate analysis to assess the independent influence of the parameters. Survival curves were plotted for the patients using the Kaplan-Meier method and analyzed using the log-rank test. Followed by calculation of $\mathrm{P}$ values using the chi-square test/Fisher's exact test. The values of the distinct groups were compared using the Mann-Whitney test. All tests were two-sided, and a $\mathrm{P}$ value of $<0.05$ was considered statistically significant. 
Table 1 Characteristics of patients of subxiphoid approach and right thoracic approach groups

\begin{tabular}{|c|c|c|c|}
\hline Clinical characters & $\begin{array}{c}\text { Subxiphoid } \\
\text { approach }(n=68)\end{array}$ & $\begin{array}{c}\text { Right thoracic } \\
\text { approach } n=63\end{array}$ & $\mathrm{P}$ \\
\hline Gender & & & 0.607 \\
\hline Male & 38 & 38 & \\
\hline Female & 30 & 25 & \\
\hline Age (year) & $45.24 \pm 16.8$ & $39.40 \pm 17.23$ & 0.510 \\
\hline $\begin{array}{l}\text { Maximal tumor } \\
\text { diameter }(\mathrm{cm})\end{array}$ & $3.14 \pm 0.98$ & $2.87 \pm 1.12$ & 0.156 \\
\hline \multicolumn{2}{|c|}{ MGFA clinical classification } & & 0.189 \\
\hline I & 24 & 32 & \\
\hline$\| A$ & 25 & 22 & \\
\hline IIB & 13 & 8 & \\
\hline III & 4 & 1 & \\
\hline IV & 2 & 0 & \\
\hline \multicolumn{2}{|c|}{ Pathological diagnoses } & & 0.151 \\
\hline Thymoma & 25 & 16 & \\
\hline $\begin{array}{l}\text { Thymic } \\
\text { hyperplasia }\end{array}$ & 34 & 40 & \\
\hline Thymic cyst & 9 & 6 & \\
\hline $\begin{array}{l}\text { Atrophy of thymus } \\
\text { gland }\end{array}$ & 0 & 1 & \\
\hline
\end{tabular}

MGFA, Myasthenia Gravis Foundation of America.

\section{Results}

\section{Clinical data of all cases}

There were 76 males and 55 females, with a median age of 46 years (range, $8-73$ years). Pathological diagnoses included thymoma $(n=41)$; thymic hyperplasia $(n=74)$; thymic cyst $(n=15)$; Atrophy of thymus gland $(n=1)$. Patient's characteristics are shown in Table 1. The clinicopathologic data including age, gender, maximal tumor diameter, body mass index (BMI), MGFA clinical classification (19), and pathological diagnoses. There is no significant difference between both groups about any of these factors.

The perioperative variables between the subxiphoid approach group and the right thoracic group are summarized in Table 2. Compared with the patients treated by conventional right thoracic approach, the duration of the procedure via subxiphoid approach was significantly shorter $(\mathrm{P}=0.035)$, with higher rates of total thymectomy
$(\mathrm{P}=0.028)$, Pain scores on postoperative days 1,3 , and 7 were significantly higher in patients who underwent right thoracic approach compared with those who underwent subxiphoid approach $(\mathrm{P}<0.001, \mathrm{P}<0.001$, and $\mathrm{P}=0.03$, respectively). No significant differences were found in blood loss during operation, Postoperative delayed extubation, postoperative crisis, Postoperative delayed extubation, postoperative hospital stay, duration, and amount of postoperative chest tube drainage among the two groups $(\mathrm{P}>0.05)$. Two patients experienced postoperative thoracotomy for hemostasis in the right thoracic approach group, and one patient was needed re-tracheal intubation after the operation in the subxiphoid approach group. Three patients experienced a conversion to thoracotomy in both groups, and the presence of tumor adhesions being the first reason for conversion without significantly statistically differences between these values. There was no postoperative mortality. All postoperative complications are listed in Table 2; postoperative complications included 3 cases of a lung infection after the operation, 1 case of postoperative chylothorax, and 1 case of temporary phrenic nerve paresis in the subxiphoid approach group and 4 cases of a lung infection after the operation, 2 cases of wound infection in the right thoracic group. The difference in the complication rate between the two groups was not significant.

\section{Prognosis}

Of the 131 patients with generalized MG treated surgically, $112(85.5 \%)$ patients were eligible for follow-up evaluation, and $19(14.5 \%)$ patients were lost to follow-up evaluation (Table 3). Three patients died during the follow-up period (1 patient in the subxiphoid approach group, and 2 patients in the right thoracic approach group); Postoperative, causes of deaths are listed in Table 4. In the subxiphoid approach group, $53(91.4 \%)$ of 58 patients were effective treatment of MG [CSR in $23(39.7 \%)$ patients, PR in $8(13.8 \%)$ patients, $\mathrm{MM}$ in $21(36.2 \%)$ patients, and I in $1(1.7 \%)$ patients], and $5(8.6 \%)$ of 59 patients were ineffective treatment of MG [U in $2(3.4 \%)$ patients, $W$ in $2(3.4 \%)$, and $E$ in 1 $(1.7 \%)$ patients. Similarly, in the right thoracic group, 45 (88.2\%) of 51 were classified as effective treatment of MG at the time of follow-up [CSR in $19(37.3 \%)$ patients, PR in $6(11.8 \%)$ patients, $\mathrm{MM}$ in $19(37.3 \%)$ patients, and I in $1(1.9 \%)$ patients], and $6(11.8 \%)$ of 53 patients were ineffective treatment of MG [U in $3(5.9 \%)$ patients, E in $2(3.9 \%)$ patients and $W$ in $1(1.9 \%)$ patients]. The 
Table 2 Comparison of variables of the subxiphoid approach versus right thoracic approach surgical groups

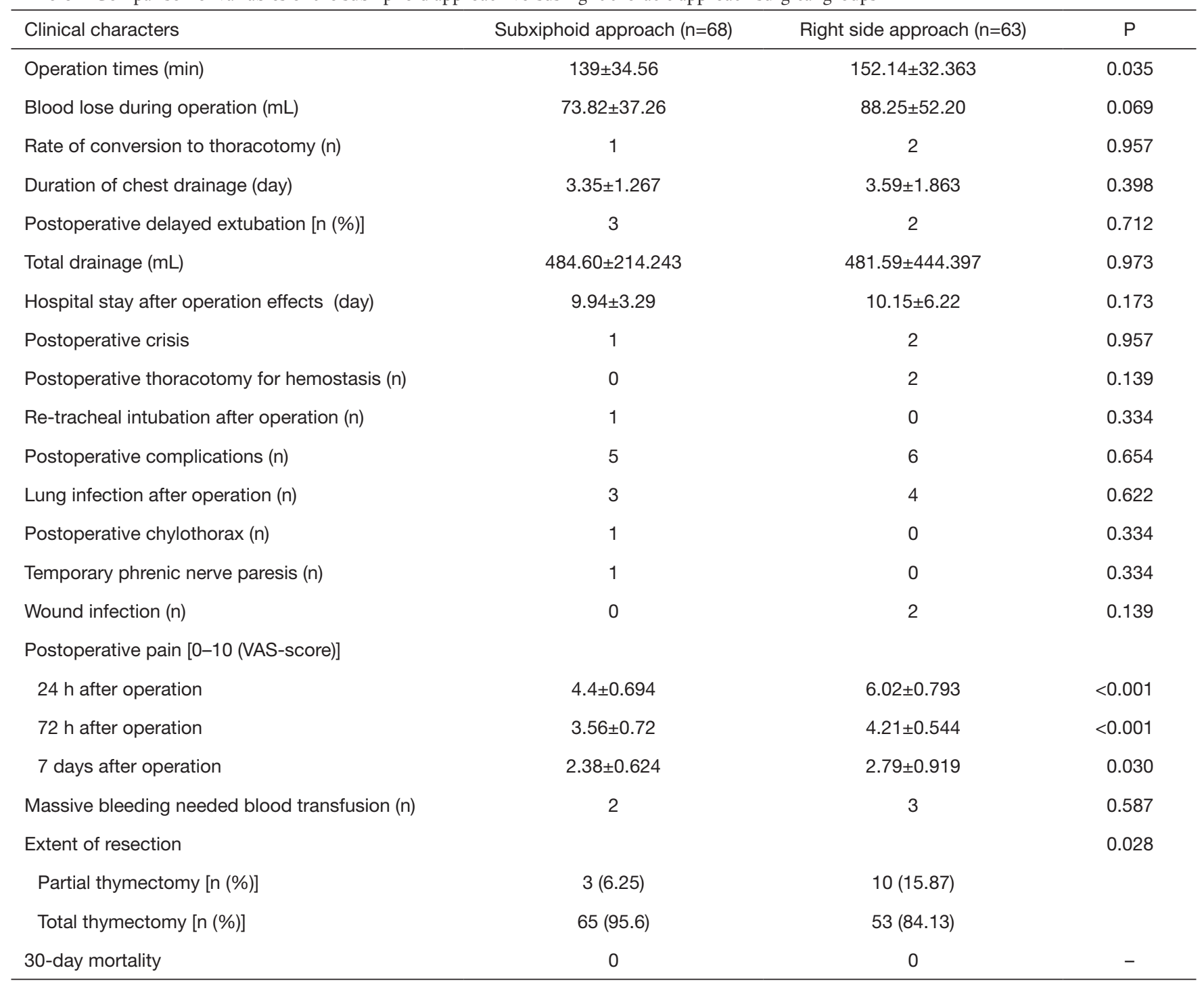

subxiphoid approach group reported higher the rates of CSR (39.7\% vs. 37.3\%) and effective treatment of MG $(91.4 \%$ vs. $88.2 \%)$ than the right-thoracic approach group, although there were no statistical differences $(Z=-0.486$, $\mathrm{P}=0.627)$. Univariate analysis showed age, pathological type, and MGFA Clinical Classification were factors affecting postoperative NCSR in patients with MG (Table 5). Multivariate analysis that age (OR: 2.502, 95\% CI: 1.169 5.357, $\mathrm{P}=0.018$ ), pathological type (OR: $1.775,95 \% \mathrm{CI}$ : 1.041-3.026, $\mathrm{P}=0.035)$ and MGFA Clinical Classification (OR: $2.024,95 \%$ CI: $1.164-3.521, \mathrm{P}=0.013$ ) were independent risk factors for postoperative treatment effect with MG (Table 6).

\section{Discussion}

In recent years, multiple minimally invasive techniques were reported for removal of the thymus in MG, the advantages of a minimally invasive approach are apparent $(12,20-22)$. The debate about which technique is preferable is not resolved. Studies by Masaoka and colleagues (23) have demonstrated that aberrant thymic tissue may occur as widely scattered foci in mediastinal fat extending to the esophagus and the diaphragm because of the high incidence of gross anatomical variations in the thymus gland. An incidence of ectopic foci of thymic tissue in the adipose tissue of the neck and the mediastinum reported by Jaretzki 
Table 3 Comparison of outcomes of myasthenia gravis after extended thymectomy

\begin{tabular}{|c|c|c|c|c|c|c|}
\hline The postintervention status & \multicolumn{2}{|c|}{ Overall $(n=109)$} & \multicolumn{2}{|c|}{ Subxiphoid approach $(n=58)$} & \multicolumn{2}{|c|}{ Right-sided approach $(\mathrm{n}=51)$} \\
\hline CSR & 42 & 38.5 & 23 & 39.7 & 19 & 37.3 \\
\hline PR & 14 & 12.8 & 8 & 13.8 & 6 & 11.8 \\
\hline MM & 40 & 36.7 & 21 & 36.2 & 19 & 37.3 \\
\hline$U$ & 5 & 4.6 & 2 & 3.4 & 3 & 5.9 \\
\hline W & 3 & 2.8 & 2 & 3.4 & 1 & 1.9 \\
\hline$E$ & 3 & 2.8 & 1 & 1.7 & 2 & 3.9 \\
\hline Effective treatment of $M G(n)^{a}$ & 98 & 89.9 & 53 & 91.4 & 45 & 88.2 \\
\hline Z & & & & -0.486 & & \\
\hline $\mathrm{P}$ & & & & 0.627 & & \\
\hline
\end{tabular}

Effects according to the American MGFA (19): CSR, complete stable remission; PR, pharmacological remission; MM, minimal manifestation; I, improved; $U$, unchanged; W, worse; E, exacerbation; MG, myasthenia gravis. ${ }^{a}, C S R+P R+M M+I ;{ }^{b}, U+W+E+D$.

Table 4 Causes of deaths of the patients operated on for MG

\begin{tabular}{llllll}
\hline No. & Sex & Age & Pathological diagnoses & Surgical approach & Cause of death \\
\hline 1 & M & 66 & Thymoma & Subxiphoid approach & Liver cancer \\
2 & M & 46 & Thymic hyperplasia & Right thoracic approach & A myasthenic crisis causes respiratory failure \\
3 & M & 49 & Thymoma & Right thoracic approach & A myasthenic crisis causes respiratory failure \\
\hline
\end{tabular}

MG, myasthenia gravis.

and Wolff (24) was $98.0 \%$, and "maximal" thymectomy was required to ensure removal of all available thymus. Although classically, "total thymectomy" is considered the goal of surgery, it has not been demonstrated unequivocally that this is necessary, nor is it clear that all the resection techniques do achieve this goal (19). Zieliński et al. (12) studies revealed ectopic foci of the thymic tissue were discovered in the fat of the neck and the mediastinum in $56.9 \%$ of patients from the extended thymectomy group, and actively support the importance of the removal of ectopic foci of the thymic tissue. Therefore, Considering the wide distribution of ectopic thymic tissues in the anterior mediastinum, most thoracic surgeons recommend to remove as much mediastinal fat tissues as possible during thymectomy to avoid leaving ectopic thymic tissues, the necessity of performing a thymectomy with an extended technique. The so-called extended thymectomy refers to the removal of the suspicious ectopic thymus as much as possible to achieve "complete resection."

Thus far, the unilateral approach has been considered as a standard procedure of VATS thymectomy. The unilateral approach for thoracoscopic thymectomy applies to noninvasive thymoma combined with MG. However, it is difficult to expose the contralateral side and to remove all the mediastinal fat tissues. Although the bilateral approach provided adequate exposure of the anterior mediastinum can make up for this deficiency, a higher number of incisions was needed, which may increase operative trauma and postoperative pain (8). Besides, the cosmetic result is not satisfying, especially in female patients. In 2003, a patient with ocular symptoms MG was treated by thoracoscopic thymectomy via the right thoracic approach, 
Table 5 Univariate analyses for the postoperative treatment effect (Cox proportional hazards model)

\begin{tabular}{|c|c|c|c|c|c|c|}
\hline Factor & \multicolumn{2}{|c|}{ Overalls } & OR & \multicolumn{2}{|c|}{$95 \% \mathrm{Cl}$} & $P$ \\
\hline Sex & & & & & & 0.927 \\
\hline Female & 62 & 56.9 & Ref. & & & \\
\hline Male & 47 & 43.1 & 0.927 & 0.600 & 1.593 & \\
\hline$<40$ & 37 & 33.9 & Ref. & & & \\
\hline$\geq 40$ & 72 & 66.1 & 3.033 & 1.444 & 6.371 & \\
\hline Pathological diagnoses & & & & & & 0.004 \\
\hline Nonthymoma & 72 & 66.1 & Ref. & & & \\
\hline I & 51 & 47.8 & Ref. & & & \\
\hline IIA and above & 58 & 52.2 & 2.230 & 1.294 & 3.843 & \\
\hline Extent of resection & & & & & & 0.216 \\
\hline Total thymectomy & 10 & 9.2 & Ref. & & & \\
\hline Partial thymectomy & 99 & 90.8 & 1.535 & 0.779 & 3.022 & \\
\hline Surgical approach & & & & & & 0.070 \\
\hline Subxiphoid approach & 58 & 53.2 & Ref. & & & \\
\hline Right thoracic approach & 51 & 46.8 & 0.637 & 0.391 & 1.038 & \\
\hline
\end{tabular}

Table 6 Multivariate analyses for the postoperative treatment effect (Cox proportional hazards model)

\begin{tabular}{|c|c|c|c|c|c|}
\hline Factor & SE & Wald & OR & $95 \% \mathrm{Cl}$ & $P$ \\
\hline Pathological diagnoses & 0.272 & 4.442 & 1.775 & $1.041-3.026$ & 0.035 \\
\hline MGFA clinical classification & 0.282 & 6.234 & 2.024 & $1.164-3.521$ & 0.013 \\
\hline Surgical approach & 0.269 & 1.841 & 0.695 & $0.410-1.176$ & 0.175 \\
\hline
\end{tabular}

MGFA, Myasthenia Gravis Foundation of America.

with left lobe thymus retained. After six years, the patient developed into generalized muscle weakness and underwent reoperation, the postoperative pathological diagnosis of thymic hyperplasia. We recently changed the approach of VATS thymectomy from the lateral approach to the subxiphoid approach. Although the subxiphoid approach has been previously reported. The subxiphoid video-assisted thoracoscopic thymectomy was first reported by Akamine et al. (25). in 1999, and then Hsu et al. (14) and Zieliński et al. (26) reported thoracoscopic thymectomy partially or only via subxiphoid incisions. Compared with the lateral VATS approach. Firstly, this technique can achieve a surgical field of vision, like mediastinal thoracotomy. An artificial pneumothorax was created by insufflation of a $10-12 \mathrm{mmHg}$ positive pressure $\mathrm{CO}_{2}$, which enabled enlargement of the retrosternal space, we were able to achieve a working space to resect the thymus and obtain a better view of the bilateral phrenic nerves and the upper area of the innominate vein 
(Figure 1). All the possible thymic bearing mediastinal fat tissues can be removed under direct thoracoscopic view, which may later translate into better results. Besides, this method does not pass through the intercostal and sternum. By avoiding intercostals nerve compression and injury, postoperative pain is significantly relieved with no risk of disruption or infection of the sternotomy wound. Zhao et al. (27) reported 13 cases of the three-hole methods of thoracoscopic thymectomy with the subxiphoid and subcostal approach. It is believed that the partial thymoma tissue invasive pericardium, lung tissue, and left innominate vein of Masaoka III thymoma can also altogether remove the tumor and thoroughly clean the fat. This procedure has the advantage of being minimally invasive and supplies an excellent view of the anterior mediastinum, allowing the surgeon to perform total thymectomy with resection of the perithymic fat. Therefore, the thoracoscope of the subxiphoid approach in the thymectomy has reduced the trauma of the operation and pursued the maximum extent of thymectomy.

Our results showed that there was no statistically significant difference between the complete remission rate and the effective rate in both groups. In the subxiphoid approach group, the operation time was shorter than that of the right thoracic approach group, and there was lower postoperative pain. This is similar to the results reported at home and abroad $(28,29)$. One patient with obesity was attached to the surrounding tissue due to dense adhesion of the thymus, and the thymus surrounded the innominate vein. The separation was difficult. For the sake of surgical safety and the principle of tumor treatment, the "J" incision of the upper sternum was completed. Only 1 patient in the subxiphoid approach group underwent re-tracheal intubation operation. No serious postoperative complications occurred in two groups. Following these data, younger patients (less than 40 years of age), non-thymomatous MG and type I (MGFA Clinical Classification) had a higher complete remission rate after thymectomy in our trial. We were able to confirm that the surgical approach is not a prognostic factor in MG.

After an extended period of research, we realized the advantages of the subxiphoid approach: first, the patient's supine position, single-lumen endotracheal intubation, easy to operate. A double-lumen tracheal tube or blocking the main bronchus was not necessary; second, many surgeons have increased the working spaces by lifting of the sternum. Combined with artificial pneumothorax, it can obtain a surgical field like that of the median sternotomy.
The operation space is wide; the looking-up view from the main incision using the subxiphoid approach was familiar to the thoracic surgeons. The thymus gland and its surrounding adipose tissue can be removed entirely. The bilateral phrenic nerves are exposed to avoid damage and to minimize the chance of accidental surgical injury; third, postoperative pain is significantly relieved, and no chest wall paresthesia is abnormal. The intercostal nerve compression or injury caused postoperative pain. Also, a postoperative incision is more beautiful and has a better choice for young patients. Therefore, the subxiphoid approach to thoracoscopic surgery in the thymectomy has reduced the trauma of the surgery itself while pursuing the maximum extent of thymectomy. However, there are some disadvantages to this procedure: Firstly, patients with obese body size and large tumors $(>8 \mathrm{~cm}$ in diameter, according to our experience, the largest tumor resected through this approach was $8 \mathrm{~cm}$ in longer diameter) should be carefully selected for this surgical approach because even if positive pressure $\mathrm{CO}_{2}$ is used to expand the space, the space of the anterior mediastinum It is also limited; Second, the anterior mediastinal operation space is relatively small, the surgical instruments are easy to interfere with each other, and the assistants are required to be high, and need to cooperate with the surgeons; Third, for patients with poor heart function, use this method with caution, because the heart compression caused by the removal of the left pericardium fat may lead to slow heart rate and even cardiac arrest. Therefore, patients with poor heart function require comprehensive monitoring during adopting this approach.

Its retrospective single-center design limited the present study. The surgical procedure performed and the decision to convert to open surgery were at the discretion of the surgeon, which might have resulted in choice bias. Our series had a limited number of cases, and the follow-up duration was not long enough. More studies with more significant numbers of patients and ten years of follow-up must evaluate the long-term survival and disease-free rates. Besides, the nature of retrospective research cannot avoid the inherent bias. Therefore, it is necessary to conduct a propensity ratio analysis or a prospective study in the future.

\section{Conclusions}

In summary, the results of the present study have shown that the subxiphoid approach VATS thymectomy is safe and possible for the treatment of MG patients, and the midterm results are promising. Furthermore, the subxiphoid 
approach is the better choice if extended thymectomy is the primary concern.

\section{Acknowledgments}

Funding: This study was partially supported by the Provincial Health Commission of Jiangxi province (No. 20195044).

\section{Footnote}

Conflicts of Interest: All authors have completed the ICMJE uniform disclosure form (available at http://dx.doi. org/10.21037/jtd.2020.03.43). The authors have no conflicts of interest to declare.

Ethical Statement: The authors are accountable for all aspects of the work in ensuring that questions related to the accuracy or integrity of any part of the work are appropriately investigated and resolved. The Institutional Review Board approved the study of the hospital (No. 2013086), and written informed consent from each patient was obtained before the procedure.

Open Access Statement: This is an Open Access article distributed in accordance with the Creative Commons Attribution-NonCommercial-NoDerivs 4.0 International License (CC BY-NC-ND 4.0), which permits the noncommercial replication and distribution of the article with the strict proviso that no changes or edits are made and the original work is properly cited (including links to both the formal publication through the relevant DOI and the license). See: https://creativecommons.org/licenses/by-nc-nd/4.0/.

\section{References}

1. Lu Q, Zhao J, Wang J, et al. Subxiphoid and subcostal arch "Three ports" thoracoscopic extended thymectomy for myasthenia gravis. J Thorac Dis 2018;10:1711-20.

2. Rückert JC, Walter M, Muller JM. Pulmonary function after thoracoscopic thymectomy versus median sternotomy for myasthenia gravis. Ann Thorac Surg 2000;70:1656-61.

3. Manoly I, Whistance RN, Sreekumar R, et al. Early and mid-term outcomes of trans-sternal and video-assisted thoracoscopic surgery for thymoma. Eur J Cardiothorac Surg 2014;45:e187-93.

4. Meyer DM, Herbert MA, Sobhani NC, et al. Comparative Clinical Outcomes of Thymectomy for Myasthenia Gravis
Performed by Extended Transsternal and Minimally Invasive Approaches. Ann Thorac Surg 2009;87:385-90; discussion 390-1.

5. Chen H, Xu G, Zheng W, et al. Video-assisted thoracoscopic extended thymectomy using the subxiphoid approach. J Vis Surg 2016,2:157.

6. Shigemura N, Shiono H, Inoue $M$, et al. Inclusion of the transcervical approach in video-assisted thoracoscopic extended thymectomy (VATET) for myasthenia gravis: a prospective trial. Surg Endosc 2006;20:1614-8.

7. Hsu CP, Chuang CY, Hsu NY, et al. Comparison between the right side and subxiphoid bilateral approaches in performing video-assisted thoracoscopic extended thymectomy for myasthenia gravis. Surg Endosc 2004;18:821-4.

8. Tamura M, Shimizu Y, Hashizume Y. Pain following thoracoscopic surgery: retrospective analysis between single-incision and three-port video-assisted thoracoscopic surgery. J Cardiothorac Surg 2013;8:153.

9. Zieliński M, Rybak M, Wilkojc M, et al. Subxiphoid video-assisted thorascopic thymectomy for thymoma. Ann Cardiothorac Surg 2015;4:564-6.

10. Zhao J, Wang J, Zhao Z, et al. Subxiphoid and subcostal arch thoracoscopic extended thymectomy: a safe and feasible minimally invasive procedure for selective stage III thymomas. J Thorac Dis 2016;8:S258-64.

11. Murai H, Uchiyama A, Mei F, et al. Long-term effects of infrasternal mediastinoscopic thymectomy in myasthenia gravis. J Neurol Sci 2009;287:185-7.

12. Zieliński M, Kużdżał J, Szlubowski A, et al. Comparison of late results of basic transsternal and extended transsternal thymectomies in the treatment of myasthenia gravis. Ann Thorac Surg 2004;78:253-8.

13. Kido T, Hazama K, Inoue $Y$, et al. Resection of anterior mediastinal masses through an infrasternal approach. Ann Thorac Surg 1999;67:263-5.

14. Hsu CP, Chuang CY, Hsu NY, et al. Subxiphoid approach for video-assisted thoracoscopic extended thymectomy in treating myasthenia gravis. Interact Cardiovasc Thorac Surg 2002;1:4-8.

15. Otsuka R, Ueda K, Tanaka T, et al. Who will benefit from thymectomy for myasthenia gravis? Is there any role for this procedure in elderly patients? Ann Transl Med 2019;7:4.

16. Nam TS, Lee SH, Kim BC, et al. Clinical characteristics and predictive factors of myasthenic crisis after thymectomy. J Clin Neurosci 2011;18:1185-8.

17. Watanabe A, Watanabe T, Obama T, et al. Prognostic 
factors for myasthenic crisis after transsternal thymectomy in patients with myasthenia gravis. J Thorac Cardiovasc Surg 2004;127:868-76.

18. Wendell LC, Levine JM. Myasthenic Crisis. Neurohospitalist 2011;1:16-22.

19. Jaretzki A 3rd, Barohn RJ, Ernstoff RM, et al. Myasthenia gravis: recommendations for clinical research standards. Task Force of the Medical Scientific Advisory Board of the Myasthenia Gravis Foundation of America. Ann Thorac Surg 2000;70:327-34.

20. Calhoun RF, Ritter JH, Guthrie TJ, et al. Results of transcervical thymectomy for myasthenia gravis in 100 consecutive patients. Ann Surg 1999;230:555-9; discussion 559-61.

21. Gonzalez-Rivas D, Paradela M, Fieira E, et al. Singleincision video-assisted thoracoscopic lobectomy: initial results. J Thorac Cardiovasc Surg 2012;143:745-7.

22. Murai H, Uchiyama A, Mei F, et al Long-term effects of infrasternal mediastinoscopic thymectomy in myasthenia gravis. J Neurol Sci 2009;287:185-7.

23. Masaoka A, Nagaoka Y, Kotake Y. Distribution of thymic tissue at the anterior mediastinum. Current procedures in thymectomy. J Thorac Cardiovasc Surg 1975;70:747-54.

Cite this article as: Qiu Z, Chen L, Lin Q, Wu H, Sun H, Zhou X, Hu Y, Peng L, Liu Y, Xu Q. Perioperative outcomes and mid-term effects in performing video-assisted thoracoscopic extended thymectomy for myasthenia gravis: subxiphoid versus right thoracic approaches. J Thorac Dis 2020;12(4):1529-1539. doi: $10.21037 /$ jtd.2020.03.43
24. Jaretzki A 3rd, Wolff M. "Maximal" thymectomy for myasthenia gravis. Surgical anatomy and operative technique. J Thorac Cardiovasc Surg 1988;96:711-6.

25. Akamine S, Takahashi T, Oka T, et al. Thymic cystectomy through subxyphoid by video-assisted thoracic surgery. Ann Thorac Surg 1999;68:2339-41.

26. Zieliński M, Kuzdzał J, Szlubowski A, et al. Transcervicalsubxiphoid-videothoracoscopic "maximal" thymectomy-operative technique and early results. Ann Thorac Surg 2004;78:404-9; discussion 409-10.

27. Zhao J, Wang J, Zhao Z, et al. Subxiphoid and subcostal arch thoracoscopic extended thymectomy: a safe and feasible minimally invasive procedure for selective stage III thymomas. J Thorac Dis 2016;8:S258-64.

28. Yano M, Moriyama S, Haneda H, et al. The Subxiphoid Approach Leads to Less Invasive Thoracoscopic Thymectomy Than the Lateral Approach. World J Surg 2017;41:763-70.

29. Suda T, Hachimaru A, Tochii D, et al. Video-assisted thoracoscopic thymectomy versus subxiphoid singleport thymectomy: initial results. Eur J Cardiothorac Surg 2016;49:i54-8. 